\title{
Hematopoietic Chimera in a Male Blood Donor and His Dizygotic Twin Sister
}

\author{
Marcos P. Miola ${ }^{a}$ Alessandro G. Lopes ${ }^{\mathrm{b}}$ Alessandra P. Silva ${ }^{c}$ \\ Edney G.C. Gomes ${ }^{c}$ Leticia A.F. Machado ${ }^{c}$ Wanessa A. Veloso ${ }^{c}$ \\ Carlos A. Costa ${ }^{c}$ Roberta M. Fachini ${ }^{d}$ Octávio Ricci Junior ${ }^{d}$ \\ Cinara C. Brandão de Mattos ${ }^{a}$ Luiz Carlos de Mattos ${ }^{a}$ \\ aDepartment of Molecular Biology, Medical School of São José do Rio Preto (FAMERP), São José do Rio Preto, Brazil; \\ ${ }^{b}$ Department of Biology, Instituto de Biociências, Letras, Ciências Exatas (IBILCE), Campus de São José do Rio Preto, \\ São José do Rio Preto, Brazil; ' Department of Special Services, Hemotherapy Service, Marinha do Brasil, Hospital

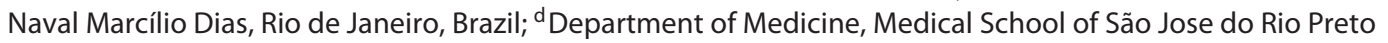 \\ (FAMERP), São José do Rio Preto, Brazil
}

\author{
Keywords \\ Chimerism · Dizygotic twins · Blood groups · Phenotyping · \\ Molecular analysis
}

\begin{abstract}
Twin hematopoietic chimera in humans is a phenomenon that was discovered accidentally and the prevalence of which remains unclear. The resolution of chimera cases requires studying family medical records, data analysis, and investigations of hematopoietic cells and cells from other tissues. The interactions among ABO, Lewis, and secretor histo-blood group systems are explored to resolve cases of hematopoietic chimera. Here we report a rare case of hematopoietic chimera where twins present a mixed field reaction in the $A B O, R h$, and Kidd red blood cell phenotyping. Using red blood cells separated from the mixed field as well as molecular approaches and investigations of family members, we identify inconsistent genotypes with the Mendelian inheritance pattern when comparing the peripheral blood with the buccal epithelium of the male twin and his twin sister. Analysis of the ABO, Lewis, and secretor phenotypes, and genomic DNA from buccal epithelium showed the genotypes $A B O^{*} A 1.01 / A_{B O}^{*} B .01$ and FUT2*01N.02/ FUT2*01N.02 in the male twin and the genotypes $A B O * 0.01 .01 / A B O * 0.01 .02$ and FUT2*01/FUT2*01 in the fe-
\end{abstract}

male twin. The results of the HLA-DRB1 genotyping showed inconsistency between the male and his twin sister. We conclude that the serological analyses combined with molecular approaches used in this study are good tools to resolve cases of hematopoietic chimera.

(c) 2019 S. Karger AG, Basel

\section{Introduction}

Hematopoietic chimerism is characterized by the presence of two populations of genetically distinct cells in the same individual, originating from different zygotes [1]. Twin hematopoietic chimerism is rarely described and sometimes presents a mixed field (MF) reaction in serological blood typing if the twins differ by distinct red blood cell (RBC) antigens from different blood group systems [2].

When hematopoietic chimerism occurs, it might allow an incorrect interpretation of the blood group phenotypes in blood donors, especially when the chimeric cells are present in small proportions. Besides, the presence of small quantities of genomic DNA containing one specific $A B O$ allele coexisting with other $A B O$ alleles might not be detected $[3,4]$. Consequently, it increases the risk of a hemolytic reaction in recipients depending on the volume of chimeric incompatible RBCs transfused [2,5].

\section{KARGER}

(C) 2019 S. Karger AG, Basel 
a

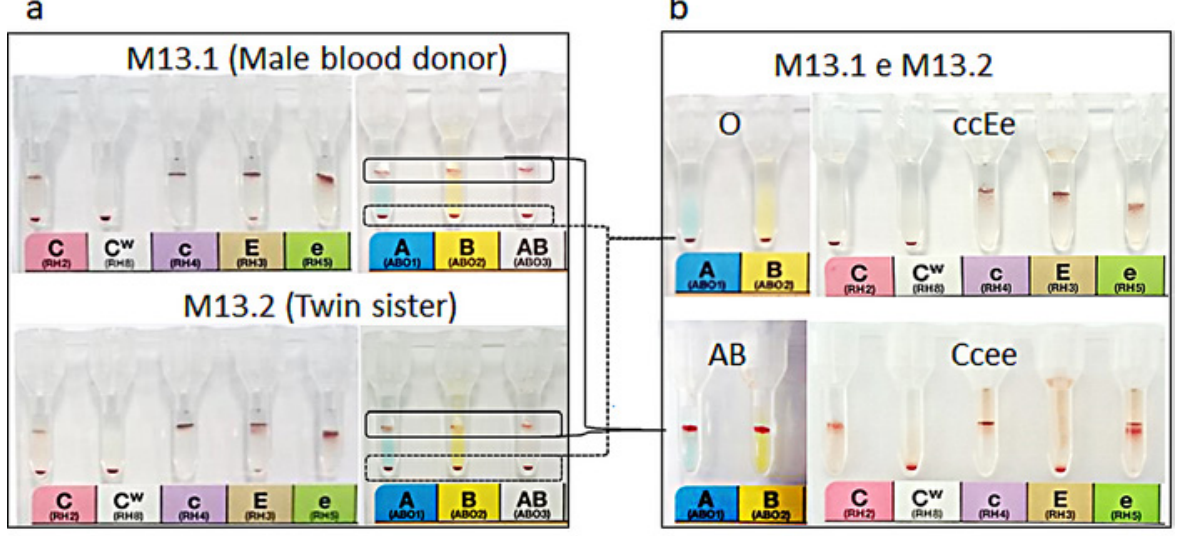

Fig. 1. a Mixed field (MF) for $A B O$ and Rh in both the male blood donor and his twin sister. b $\mathrm{ABO}$ and $\mathrm{Rh}$ phenotypes after cell separation from the MF, demonstrating that the male blood donor has an $\mathrm{AB}$ phenotype and his twin sister has an $\mathrm{O}$ phenotype. c ABO and Rh phenotypes from both parents.

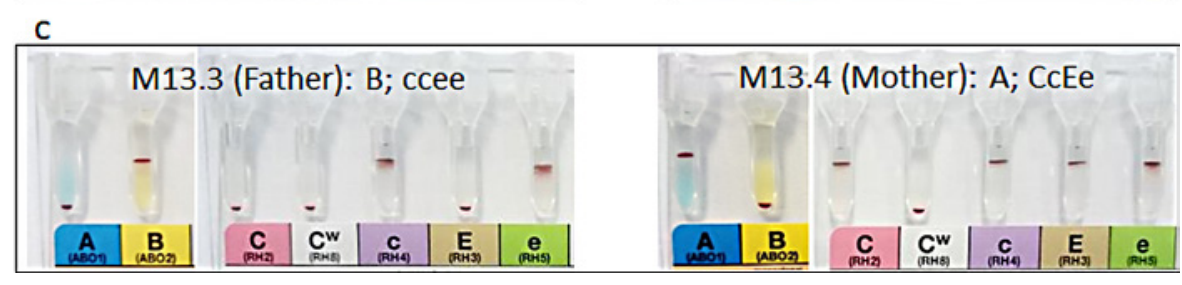

Many reports describing hematopoietic chimerism in twins have been published. In one of them, Lee et al. [6] describe a case where the twins, one male and the other female, were classified as a B3 subgroup at birth. Subsequent serological analysis showed that they were from the $\mathrm{B}$ phenotype but presenting MF reaction in the forward phenotyping. Molecular analysis revealed the presence of three $A B O$ alleles $\left(A B O^{*} B .01, A B O^{*} O .01 .01\right.$, and $\left.A B O^{*} O .01 .02\right)$ in both twins. In the other report, Sharpe et al. [7] introduced a case presenting $\mathrm{MF}$ reaction in the $\mathrm{ABO}, \mathrm{RhD}$, and $\mathrm{RhE}$ phenotyping. They evaluated the FUT2 gene to define the secretor phenotype since the case presented a small proportion of A RBCs that reacted weakly with anti-A antisera. Yang et al. [4] reported a case showing MF reaction with anti-A but presenting anti-B antibody in the reverse phenotyping. Exploring a careful review of a sequence electropherogram, they were able to identify three $A B O$ alleles $\left(A B O^{*} 0.01 .01, A B O^{*} O .01 .02\right.$, and $\left.A B O^{*} A 1.02\right)$.

These studies, among others [8-10], demonstrate that the resolution of hematopoietic chimerism between twins is complex and should combine serological and molecular investigations of genes, such as $A B O$ and FUT2, as well as studies in tissues other than hematopoietic, and in other family members. The aim of this study was to resolve a case of hematopoietic chimerism where dizygotic twins presented an MF reaction in the forward $\mathrm{ABO}$ phenotyping.

\section{Materials and Methods}

\section{Case Description}

A male blood donor (M13.1) and his twin sister (M13.2) both presenting an $\mathrm{MF}$ reaction in the $\mathrm{ABO}$ forward phenotyping, with the absence of plasma regular anti-A and anti-B antibodies, were referred to our laboratory for serological and molecular investigation. Both M13.1 and M13.2, with no history of previous immunological abnormalities, presented a proportion of nonagglutinated RBCs apparently higher than agglutinated RBCs by anti-A, anti-B, and anti-A,B antisera in the gel column (Fig. 1a). MF was also observed for some Rh antigens $(\mathrm{C}, \mathrm{E})$ as well as for Kidd $\left(\mathrm{Jk}^{\mathrm{a}}\right)$ but not for Lewis systems using commercial gel columns (DiaMed Latin America, Brazil). The other family members (father, mother, and non-twin sister) were also studied (Fig. 1c; Table 1). Extended serological and molecular investigations were performed only for $\mathrm{ABO}$ and secretor phenotypes.

\section{Serological Investigation}

The ABO forward and reverse phenotyping was carried out in tubes and gel columns (DiaMed Latin America) with the following commercial antibodies: anti-A (clones: LC A5, 9113D10, and LM 297/628 [LA-2]), anti-B (clones: LC G1/2, 9621 A8, and LM 306/686 [LB-2]), and anti-A,B (clones: LC Birna-1, ES-4, ES131 [ES15], and $9113 \mathrm{D} 10+152 \mathrm{D} 12)$. Commercial anti-A1 (Dolichos biflorus) and anti-H (Ulex europaeus) were also used.

The secretor and nonsecretor phenotypes were determined from saliva with the same antibodies mentioned above. Lewis phenotypes of RBCs and saliva were performed in tubes with the anti$\mathrm{Le}^{\mathrm{a}}$ (clone: LEA2 e3643B9) and anti-Le ${ }^{\mathrm{b}}$ (clone: LEB2 GX336) commercial antisera (Lorne Laboratories, USA; Fresenius-Kabi, Brazil).

The MF RBCs were separated as suggested by Svensson et al. [8] with adaptation, using a pipette, gel column neutral cards, tubes, and commercial ABO antibodies. Firstly, the agglutinated RBCs were removed and then treated with $0.2 \mathrm{M}$ dithiothreitol (DTT) and phenotyped. Secondly, the nonagglutinated RBCs were transferred to tubes, washed in saline solution, and phenotyped. The RBCs from both twins were not pooled and the phenotyping procedures were carried out in separate gel columns (Fig. 1a, b).

\section{Molecular Genotyping of ABO and FUT2 Genes}

Genomic DNA from all individuals was extracted from peripheral blood using commercial kits (QIAamp DNA Blood Mini Kit, 
Table 1. Serological data of the male blood donor (M13.1.), his twin sister (M13.2), and other family members

\begin{tabular}{|c|c|c|c|c|c|c|c|c|c|c|c|c|c|c|c|c|}
\hline \multirow[t]{3}{*}{ Cases } & \multicolumn{5}{|c|}{$\mathrm{ABO}$} & \multicolumn{2}{|l|}{ Lectins } & \multirow{3}{*}{$\frac{\mathrm{ABH} / \text { Lewis }}{\text { saliva }}$} & \multirow[t]{3}{*}{ Lewis } & \multicolumn{4}{|l|}{$\mathrm{Rh}$} & \multicolumn{2}{|c|}{ Kidd } & \multirow{3}{*}{$\begin{array}{l}\text { Phenotyping after } \\
\text { RBC separation of } \\
\text { the MF }\end{array}$} \\
\hline & \multicolumn{3}{|c|}{ forward } & \multicolumn{2}{|c|}{ reverse } & \multirow[t]{2}{*}{ anti-A1 } & \multirow[t]{2}{*}{ anti-H } & & & \multirow[t]{2}{*}{$\mathrm{C}$} & \multirow[t]{2}{*}{ c } & \multirow[t]{2}{*}{$\mathrm{E}$} & \multirow[t]{2}{*}{$\overline{\mathrm{e}}$} & \multirow[t]{2}{*}{$\mathrm{Jk}^{\mathrm{a}}$} & \multirow[t]{2}{*}{$\mathrm{Jk}^{\mathrm{b}}$} & \\
\hline & A & B & $\mathrm{AB}$ & A1 & B & & & & & & & & & & & \\
\hline M13.1 (blood donor) & MF & MF & MF & 0 & 0 & MF & 2 & $\mathrm{Le}^{\mathrm{a}}$ & $\operatorname{Le}(a+b-)$ & MF & 4 & MF & 4 & MF & 4 & $\mathrm{AB} ; \mathrm{Ccee} ; \mathrm{Jk}(\mathrm{a}+\mathrm{b}+)$ \\
\hline M13.2 (twin sister) & MF & MF & MF & 0 & 0 & MF & 2 & $\mathrm{H}, \mathrm{Le}^{\mathrm{a}}, \mathrm{Le}^{\mathrm{b}}$ & $\operatorname{Le}(a-b+)$ & MF & 4 & MF & 4 & MF & 4 & $\mathrm{O} ; \mathrm{ccEe} ; \mathrm{Jk}(\mathrm{a}-\mathrm{b}+)$ \\
\hline M13.3 (father) & 0 & 4 & 4 & 4 & 0 & 0 & 2 & NT & $\operatorname{Le}(a-b+)$ & 0 & 4 & 0 & 4 & 4 & 4 & - \\
\hline M13.4 (mother) & 4 & 0 & 4 & 0 & 4 & 3 & 1 & NT & $\operatorname{Le}(a-b+)$ & 4 & 4 & 4 & 4 & 0 & 4 & - \\
\hline M13.5 (non-twin sister) & 4 & 4 & 4 & 0 & 0 & 3 & 1 & NT & $\operatorname{Le}(a+b-)$ & 4 & 4 & 0 & 4 & 4 & 4 & - \\
\hline
\end{tabular}

MF, mixed field; NT, not tested.

QIAGEN, Hilden, Germany). Genomic DNA from the buccal epithelium (oral swab) was extracted with a phenol-chloroform method [9].

We developed a conventional PCR protocol using six primers (M5-ABOex6-F: 5'-AGCTGAGTGGAGTTTCCAGGT-3'; M6ABOex6-R: 5'-GAAGGAGCTGGGTTTTACCG-3'; M5-ABOex7 -F: 5'-AGGACTCGCTCAGGACAGG-3'; M8-ABOex7-R: $5^{\prime}$ CTTGTTCAGGTGGCTCTCGT-3'; ${ }^{\prime}$ M7-ABOex7-F: $5^{\prime}$-CTTCCTCAGCGAGGTGGATT-3'; M4-ABOex7-R: 5'-GGACGGACAAAGGAAACAGA- $3^{\prime}$ ) to evaluate exons 6 and 7 of the $A B O$ gene by sequencing. The primers M5-ABOex6-F and M6-ABOex6-R were also used to identify the main polymorphism of the $O$ alleles (261delG) by PCR-RFLP [10].

Four primers (F1-FUT2ex2-F: 5'-CCTGTGCACATAGGCAAGTATG-3'; F2-FUT2ex2-R: 5'-CACCCCCTTCCACACTTTTG-3'; F3-FUT2ex2-F: 5'-AACGACTGGATGGAGGAGGA-3'; F4-FUT2ex2-R: $5^{\prime}$-CAGGCCACTGTTCACTGAGATT-3') were used to evaluate exon 2 of the FUT2 gene by sequencing. Primers F1-FUT2ex2-F and F2-FUT2ex2-R were also used to identify the main polymorphism of the FUT2 gene $(428 \mathrm{G}>\mathrm{A}, \mathrm{rs} 601338)$ by PCR-RFLP [11].

The PCR conditions were: initial denaturation $\left(94^{\circ} \mathrm{C}\right.$ for 3 min), 35 cycles of denaturation $\left(94^{\circ} \mathrm{C}, 45 \mathrm{~s}\right)$ and annealing/extension $\left(61^{\circ} \mathrm{C}, 30 \mathrm{~s} ; 72^{\circ} \mathrm{C}, 90 \mathrm{~s}\right)$, final extension $\left(72^{\circ} \mathrm{C}, 10 \mathrm{~min}\right)$, using recombinant Taq DNA polymerase (Invitrogen, Brazil). The amplified fragments were purified with a commercial GenElute PCR Clean-Up Kit (Sigma-Aldrich Brazil). The sequencing analysis was performed using commercial kits (BigDye Terminator, Applied Biosystems, Foster City, CA, USA) on an ABI PRISM 3500 Genetic Analyzer (Applied Biosystems). The $A B O$ and FUT2 sequences obtained were aligned with the NCBI reference sequence for $A B O^{*} 01.01$ (NG_006669.1) and FUT2*01 (NG_007511.1) using the BioEdit software [12].

The terminology used throughout this report follows the International Society of Blood Transfusion (ISBT; www.isbtweb.org). The SNPs c.261delG and c.297G>A from exon 6 and the SNPs c. $526 \mathrm{C}>\mathrm{G}, \mathrm{c} .646 \mathrm{~T}>\mathrm{A}, \mathrm{c} .657 \mathrm{C}>\mathrm{T}, \mathrm{c} .681 \mathrm{G}>\mathrm{A}, \mathrm{c} .703 \mathrm{G}>\mathrm{A}, \mathrm{c} .771 \mathrm{C}>\mathrm{T}$, c. $796 \mathrm{C}>\mathrm{A}$, c. $803 \mathrm{G}>\mathrm{C}, \mathrm{c} .829 \mathrm{G}>\mathrm{A}$, and c.930G $>\mathrm{A}$ at exon 7 were eligible to differentiate the $A, B$, and $O$ alleles.

\section{HLA-DRB1 Genotyping}

HLA-DRB1 genotyping was carried out by PCR-rSSO with an rSSO Luminex genotyping kit (One Lambda, Canoga Park, CA, USA) with low/medium resolution. The hybridization was checked by flow cytometry (LABScanTM100 flow analyzer; One Lambda), and the data were interpreted by HLA 2.0 Fusion Research software (One Lambda).

\section{Results}

The results of the serological analyses are shown in Table 1. The MF reaction in both M13.1 and M13.2 for ABO and $\mathrm{Rh}$ phenotypes shows apparently more quantities of nonagglutinated than agglutinated RBCs with anti-A, anti-B, anti-A, B, and anti-C. The opposite was observed for anti-E (Fig. 1a). The MF reaction was not observed in the RBC Lewis phenotyping. M13.1 and M13.2 were phenotyped as $\mathrm{Le}(\mathrm{a}+\mathrm{b}-)$ and $\mathrm{Le}(\mathrm{a}-\mathrm{b}+)$, respectively. Analysis of the saliva of M13.1 showed only Lea antigens but M13.2 secreted H, Lea, and Leb (Table 1). After the separation of RBCs, no MF in ABO and Rh phenotyping was observed for both M13.1 and M13.2 (Fig. 1b).

Molecular analysis by PCR-RFLP and sequencing using genomic DNA extracted from peripheral blood suggested the presence of more than two alleles for $A B O$ (Fig. 2) and FUT2 genes, in both M13.1 and M13.2. The analyses of the genomic DNA from oral swabs showed that M13.1 has AB and nonsecretor phenotypes, and M13.2 has $\mathrm{O}$ and secretor phenotypes. These data from $A B O$ genotyping for M13.1 and M13.2 are in accordance with those obtained from parental genotypes. The data from FUT2 genotyping agree with the Lewis RBC phenotyping and the secreted antigens in saliva (Fig. 3).

Extended molecular investigations by sequencing in the genomic DNA from peripheral blood for the $A B O$ and FUT2 genes from both M13.1 and M13.2 suggested the presence of four $A B O$ alleles and more than two FUT2 alleles. The sequencing of exon 6 of the genomic DNA from peripheral blood showed the sequence GGTGACC and GGTACC (heterozygosis), suggesting the presence of one $A$ and one $B$ allele as well as one $O$ allele carrying the deletion of a $\mathrm{G}$ nucleotide at position 261.

The same analysis from the buccal genomic DNA showed the sequence GGTGACC (homozygosis), which is compatible with one $A$ and one $B$ allele in M13.1. The sequence GGTACC appears in M13.2, which is compatible with the homozygosis for $O$ allele. The analysis of the additional mutations at exon 7 reinforces the presence of 


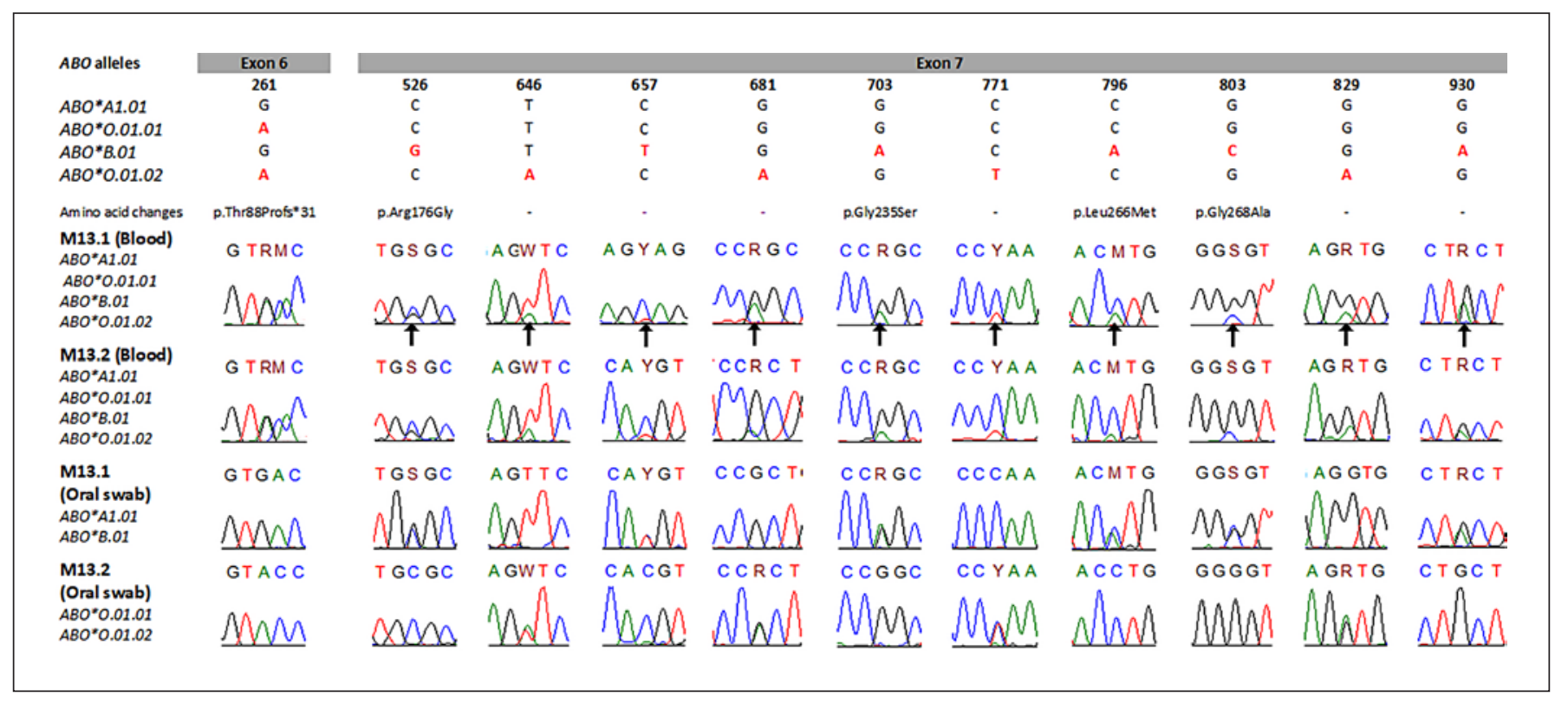

Fig. 2. The data from direct sequencing reveal small peaks in all nucleotide positions (arrows), suggesting heterozygosis in the main $A B O$ alleles $A 1, B, O .01 .01$, and 0.01 .02 in the DNA from peripheral blood. The sequencing from the DNA from oral swabs demonstrates the presence of one $A$ allele and one $B$ allele in M13.1 and two $O$ alleles in M13.2. The nomenclature R, M, S, Y, and W according to the IUPAC (International Union of Pure and Applied Chemistry) was used for double nucleotides (heterozygosis).

$A, B$, and $O$ alleles at the genomic DNA from peripheral blood from M13.1 and M13.2. On the other hand, the analysis of the oral swab genomic DNA showed the presence of one $A\left(A B O^{*} A 1.01\right)$ and one $B\left(A B O^{*} B .01\right)$ allele in M13.1 and $O$ alleles $\left(A B O^{*} O .01 .01\right.$ and $\left.A B O^{*} O .01 .02\right)$ in M13.2 (Fig. 2).

We were unable to define the HLA-DRB1 alleles for M13.1 and M13.2 from DNA extracted from peripheral blood due to ambiguities. On the other hand, the HLA$D R B 1$ genotyping showed a consistent inheritance pattern to M13.1 and M13.2 and their genitors when analyzing the oral swab genomic DNA (Fig. 3).

\section{Discussion}

In this study, we described a case of twin hematopoietic chimerism characterized by an MF reaction in the forward $\mathrm{ABO}$ phenotyping in a male blood donor and his twin sister. To verify the hematopoietic chimerism, we performed an extended serological investigation using RBCs, saliva, and oral swab (ABO, Lewis, secretor), as well as a molecular analysis of $A B O, F U T 2$, and HLA$D R B 1$ genes, using genomic DNA from hematopoietic tissue (peripheral blood) and nonhematopoietic tissue (oral swab) from the male blood donor, his twin sister, and other family members. To the best of our knowledge, this is the first case of hematopoietic chimera in a male blood donor reported in Brazil.

Hematopoietic Chimera in Dizygotic Twins
Although no quantitative analysis of RBCs has been performed by flow cytometry, it was possible to notice that both the male blood donor and his twin sister present more O RBCs than AB RBCs in the gel column. This observation suggests that he received more grafted cells from his sister than she received from him. An unbalanced placental vascular anastomosis, the diameter and the location of the vessels, as well as the blood pressure could modify the flow of cells, allowing twin chimeras to present unequal proportions of hematopoietic cells and, consequently, RBCs carrying different ABO phenotypes [13-17]. Moreover, the early exchange of cells could contribute to immune tolerance in M13.2, allowing the absence of anti-A and anti-B regular antibodies in her blood plasma. These data are in accordance with those reported by Hosoi et al. [15], who proposed that the longer the period of anastomosis between twin embryos, a greater proportion of hematopoietic cells could transfer from one to another twin.

Since the results of RBCs Lewis phenotyping from M13.1 and M13.2 were concordant with secreted ABO and Lewis antigens as well with the FUT2 genotyping, we can infer that the occurrence of a hematopoietic chimera does not modify the expression of these antigens in nonhematopoietic tissues $[16,17]$. In fact, the expression of $\mathrm{ABO}$ and Lewis antigens in nonhematopoietic tissue is under control of independent genes that are not expressed in the hematopoietic tissue $[18,19]$. For instance, Lewis antigens expressed in small intestine, pancreas, liver, and 


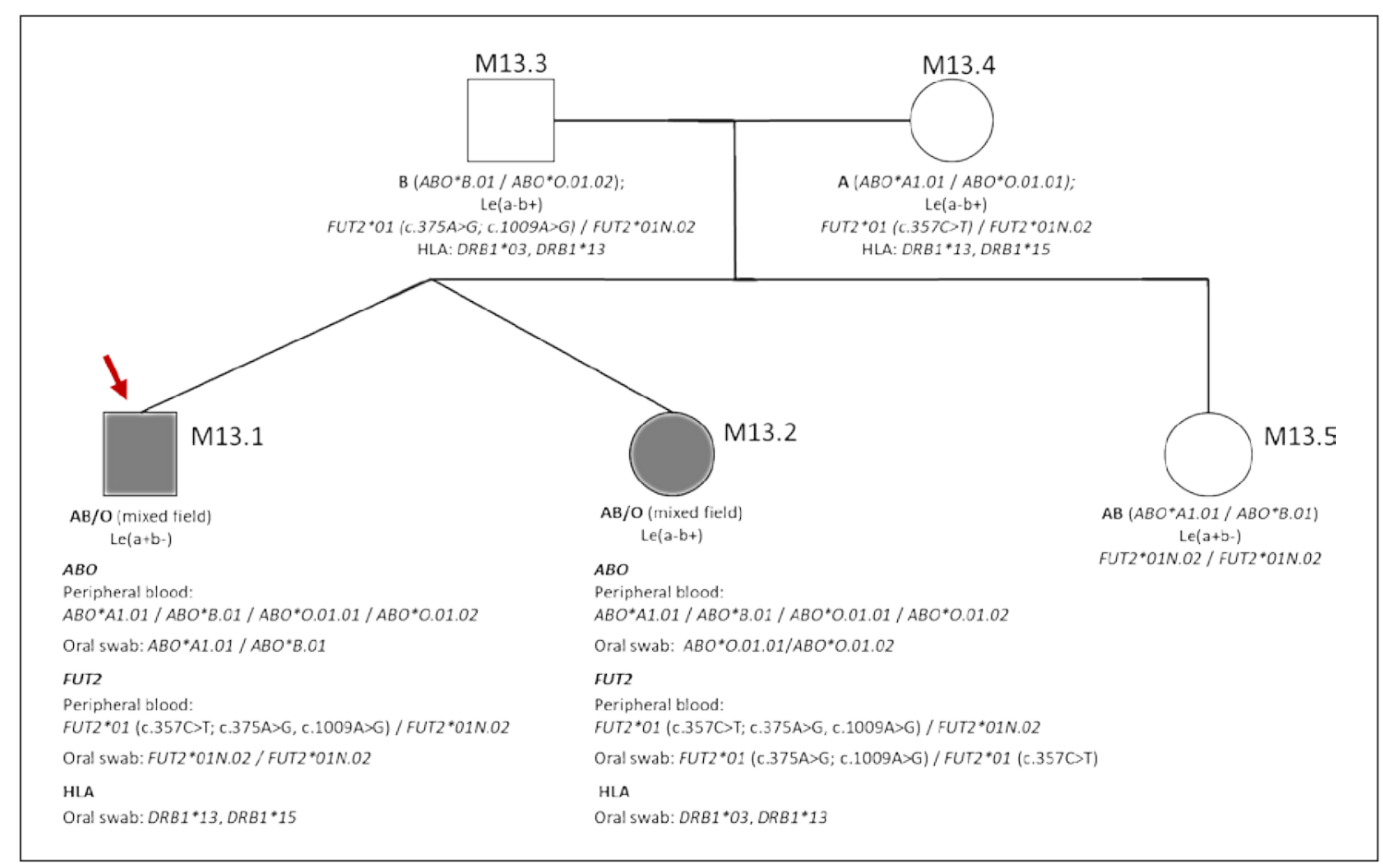

Fig. 3. Heredogram showing the phenotypes ( $A B O$ and Lewis) and genotypes ( $A B O, F U T 2$, and $H L A)$ identified in all family members.

kidney under the control of FUT2 and FUT3 genes are extrinsic to RBCs, but they are acquired from blood plasma characterizing the classical Lewis phenotypes [18].

Aiming to define this hematopoietic chimerism, we extended the serological investigation analyzing the MF RBCs separated from the gel column. This procedure allowed us to identify the phenotypes of both cell populations not just for $\mathrm{ABO}$ but also for other blood group phenotypes such as RhC, RhE, and Jk (Fig. 1b; Table 1). Therefore, the results obtained from this procedure combined with those obtained from the analysis of saliva prompted us to suggest that M13.1 has the AB phenotype and M13.2 has the O phenotype. Our interpretation is in agreement with data reported by Nicholas et al. [16], who explored RBC and saliva phenotyping of a similar case.

The presence of more than two alleles is inconsistent with the autosomal Mendelian inheritance pattern accepted for $A B O$ and FUT2 genotypes [17, 20]. This same analysis applied to genomic DNA from the father and the mother showed the presence of two $A B O$ and FUT2 alleles in each one. Although the FUT2 gene encodes for a functional fucosyltransferase able to generate $\mathrm{H}$ antigens in nonhematopoietic tissues and exocrine secretions, it has independent segregation from the $A B O$ gene due to the distinct chromosome location [21,22]. Consequently, it might express $\mathrm{H}$ antigens in nonhematopoietic tissues, which can be converted to A and or B antigens, independent of those observed on RBC surfaces $[17,19]$.
The analyses of the genomic DNA from oral swabs indicate that M13.1 has the $A B$ genotype and nonsecretor phenotype $\left(A B O^{*} A 1.01 / A B O^{*} B .01 ;\right.$ FUT2*01N.02/FUT2 $\left.{ }^{*} 01 N .02\right)$ and M13.2 has the $O$ genotype and secretor phenotype $\left(A B O^{*} 0.01 .01 / A B O^{*} 0.01 .02 ; \quad F U T 2 * 01\right.$ [c.375A $>$ G; 1009A $>$ G] $/ F U T 2 * 01$ [c.357C $>$ T] ]). This interpretation was taken from the combined results from serological and molecular analysis of their father and mother.

The comparative analysis of the genomic DNA from peripheral blood and the buccal genomic DNA from M13.1 and M13.2 suggest the presence of hematopoietic chimerism in both. This proposition is supported by the consistent results obtained from the HLA-DRB1 genotyping in the DNA extracted from buccal epithelium from M13.1, M13.2, and their parents, which are in accordance with Mendelian inheritance. Additionally, the ambiguities verified in the DNA from peripheral blood from the male and his twin sister suggest the presence of more than two HLA-DRB1 alleles in their genome. These data support the potential occurrence of hematopoietic chimerism in this case.

This study presents some limitations. Firstly, flow cytometry was not used to quantify the $\mathrm{AB}$ and $\mathrm{O}$ RBCs. Secondly, analysis by direct sequencing might be difficult to interpret, especially in situations where the amplification of the major allele can be overlooked. In fact, the careful analysis of the electropherogram showed low 
peaks in the SNPs in positions $526,657,703,796,803$, and 930 for $A B O^{*} B .01$ allele as well as in the SNPs in the positions $646,681,771$, and 829 for the $A B O^{*} 0.01 .02$ allele $[4,6]$.

Finally, the differences observed between the $A B O$ and FUT2 genotypes from genomic DNA (peripheral blood and oral swab) combined with analysis of family data, serological analysis, and with an alternative MF RBC separation method suggest that this case presents a chimerism restricted to the hematopoietic tissue. In conclusion, the male blood donor has $\mathrm{AB}$ RBCs and his twin sister has an O RBC phenotype.

\section{Acknowledgments}

The authors thank James Hesson from Academic English Solutions for their help with the English version.

\section{Statement of Ethics}

The study was approved by the Research Ethics Committee of FAMERP (CAAE 34163114.6.0000.5415). All participants were informed of the objectives of the study and signed informed-consent forms.

\section{Disclosure Statement}

The authors have no conflicts of interest to disclose.

\section{References}

1 Peters HE, König TE, Verhoeven MO, Schats R, Mijatovic V, Ket JC, et al. Unusual Twinning Resulting in Chimerism: A Systematic Review on Monochorionic Dizygotic Twins. Twin Res Hum Genet. 2017 Apr;20(2):161-8.

2 Bluth MH, Reid ME, Manny N. Chimerism in the immunohematology laboratory in the molecular biology era. Transfus Med Rev. 2007 Apr;21(2):134-46.

3 Cho D, Lee JS, Yazer MH, Song JW, Shin MG, Shin JH, et al. Chimerism and mosaicism are important causes of $\mathrm{ABO}$ phenotype and genotype discrepancies. Immunohematology. 2006;22(4):183-7.

4 Yang HS, Shin JG, Yang JJ, Choi JC, Park TS, Lee JY, et al. ABO genotype-phenotype discrepancy due to chimerism resolved by clonal separation. Ann Hematol. 2017 Sep;96(9): 1583-5.

5 Pruss A, Heymann GA, Hell A, Kalus UJ, Krausch D, Dörner T, et al. Acute intravascular hemolysis after transfusion of a chimeric RBC unit. Transfusion. 2003 Oct;43(10): 1449-51.

6 Lee OJ, Cho D, Shin MG, Kim SO, Park JT, Kim HK, et al. The first known case of blood group chimerism in monochorionic dizygotic twins in Korea. Ann Lab Med. 2014 May; 34(3):259-62.

7 Sharpe C, Lane D, Cote J, Hosseini-Maaf B, Goldman M, Olsson ML, et al. Mixed field reactions in $\mathrm{ABO}$ and $\mathrm{Rh}$ typing chimerism likely resulting from twin haematopoiesis. Blood Transfus. 2014 Oct;12(4):608-10.
8 Svensson L, Bindila L, Angström J, Samuelsson BE, Breimer ME, Rydberg L, et al. The structural basis of blood group A-related glycolipids in an A3 red cell phenotype and a potential explanation to a serological phenomenon. Glycobiology. 2011 Feb;21(2):162-74.

9 Zumbo P. Phenol-chloroform extraction. New York: Weill Cornell Medical College; 2006;1979:1-7.

10 Mattos LC, Sanchez FE, Cintra JR, et al. Genotipagem do locus ABO (9q34.1) em doadores de sangue da região noroeste do Estado de São Paulo. Rev Bras Hematol Hemoter. 2001; 23(1):15-22.

11 Svensson L, Petersson A, Henry SM. Secretor genotyping for A385T, G428A, C571T, C628T, 685delTGG, G849A, and other mutations from a single PCR. Transfusion. 2000 Jul;40(7):856-60.

12 Hall TA. BioEdit: A User-Friendly Biological Sequence Alignment Editor and Analysis Program for Windows 95/98/NT. Nucleic Acids Symp Ser. 1999;41:95-8.

13 van Dijk BA, Boomsma DI, de Man AJ. Blood group chimerism in human multiple births is not rare. Am J Med Genet. 1996 Jan;61(3): 264-8.

14 Lewi L, Deprest J, Hecher K. The vascular anastomoses in monochorionic twin pregnancies and their clinical consequences. Am J Obstet Gynecol. 2013 Jan;208(1):19-30.
15 Hosoi T, Yahara S, Kunitomo K, Saji H, Ohtsuki Y. Blood chimeric twins: an example of blood cell chimerism. Vox Sang. 1977;32(6): 339-41.

16 Nicholas JW, Jenkins WJ, Marsh WL. Human blood chimeras: a study of surviving twins. BMJ. 1957 Jun;1(5033):1458-60.

17 Daniels G. Human blood groups. 3rd ed. Oxford: Wiley; 2013.

18 Henry S, Oriol R, Samuelsson B. Lewis histoblood group system and associated secretory phenotypes. Vox Sang. 1995;69(3):166-82.

19 de Mattos LC. Structural diversity and biological importance of ABO, H, Lewis and secretor histo-blood group carbohydrates. Rev Bras Hematol Hemoter. 2016 Oct - Dec;38(4): 331-40.

20 Storry JR, Olsson ML. The ABO blood group system revisited: a review and update. Immunohematology. 2009;25(2):48-59.

21 Yamamoto F. Review: ABO blood group system-ABH oligosaccharide antigens, anti-A and anti-B, A and B glycosyltransferases, and ABO genes. Immunohematology. 2004;20(1): 3-22.

22 Marionneau S, Cailleau-Thomas A, Rocher J, Le Moullac-Vaidye B, Ruvoën N, Clément M, et al. $\mathrm{ABH}$ and Lewis histo-blood group antigens, a model for the meaning of oligosaccharide diversity in the face of a changing world. Biochimie. 2001 Jul;83(7):565-73. 